\title{
PREVALÊNCIA DE INFECÇÃO COMUNITÁRIA NA PEDIATRIA DE UM HOSPITAL PÚBLICO DA PARAÍBA
}

Prevalence of Community Infection in the Pediatrics of a Public Hospital from Paraiba

Prevalencia de infección comunitaria en pediatría de un hospital público de Paraíba

Prévalence de l'infection communautaire en pédiatrie d'un hpital public de Paraíba

Waldner Gomes Barbosa Filho1* (1); Ana Karina Holanda Leite Maia ${ }^{2}$; ; Luiz Medeiros de Araújo Lima

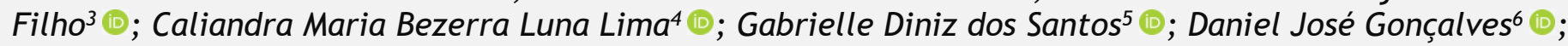
Gilberto Costa Teodózio ${ }^{7}$ (1); Raquel de Lima Pimenta ${ }^{8}$ (1)

\section{Resumo}

A infecção comunitária apesar de não ser de grande preocupação para órgãos de saúde, ela consiste em grande preocupação para os hospitais, pois podem ser risco aos pacientes já fragilizados que chegam aos serviços de saúde. Portanto, o presente estudo pretende descrever o perfil clínico e a prevalência de infecções comunitárias das crianças residentes no Vale do Mamanguape - PB, que foram internadas na pediatria do Hospital Geral de Mamanguape - PB. A metodologia trata-se de um estudo retrospectivo, de caráter exploratório, descritivo com uma abordagem quantitativa. Os dados foram extraídos dos prontuários eletrônicos e impressos de crianças internadas por um período de mais 24 horas, entre os meses de Janeiro de 2016 a Outubro de 2017, no setor da pediatria do hospital. A amostra contou com 711 crianças, de ambos os sexos, com idade de 0 ano a 15 anos. O diagnóstico de infecção comunitária foi baseado no preenchimento médico do prontuário, nos campos: motivo de internação e anamnese. Os dados foram submetidos ao software $R$, onde realizou-se a análise descritiva e inferencial dos dados. $\mathrm{Na}$ análise inferencial, aplicou-se o Teste Não-paramétrico Qui-Quadrado de Pearson afim de verificar a correlação entre a variável dependente e as variáveis fatores. Sendo assim, observou-se que $83,5 \%$ crianças foram internadas por algum tipo de infecção comunitária e $16,5 \%$ foram internados com ausência de infecção, a média de dias de internação foi de 4,94 dias $44,5 \%$ das crianças internadas foram do município de Mamanguape e $55,3 \%$ dos demais municípios que compõem o Vale do Mamanguape. As infecções mais presentes foram do trato respiratório $47,11 \%$ e gastroenterite $14,3 \%$. Crianças do sexo masculino passaram mais dias internadas do que criança do sexo feminino com $p$-valor $=0,020$. Podendo-se concluir que as infecções comunitárias são as causas mais presentes de internação na pediatria do Hospital Geral de Mamanguape-PB, as infecções do trato respiratório são as mais presentes nas crianças que residem no Vale do Mamanguape.

Palavras-chave: Diagnósticos de infecções. Infecção adquirida. Infecção em Crianças.
1 Mestre em Modelos de Decisão e Saúde, Universidade Federal da Paraíba, João Pessoa, Paraíba, Brasil.

*Corresponding author: waldnerjg@gmail.com

2 Professora Externa em graduação de Enfermagem, Universidade Federal da Paraíba, João Pessoa João Pessoa, Paraíba, Brasil.

3 Professor Colaborador do Programa de Pós Graduação em Modelos de Decisão e Saúde, Universidade Federal da Paraíba, João Pessoa, Paraíba, Brasil.

4 Professora Permanente do Programa de Pós Graduação em Modelos de Decisão e Saúde, Universidade Federal da Paraíba, João Pessoa, Paraíba, Brasil.

5 Ecóloga. Especialista em Planejamento Urbano e Gestão de Cidades (UNIPE); Mestre em Desenvolvimento e Meio ambiente (Prodema-UFPB) Paraíba, Brasil.

6 Bacharel em Biomedicina Faculde Mairicio de Nassau, Campina Grande; MBA Gestão em Saúde (USP), João Pessoa, Paraíba, Brasil.

7 Graduação em Enfermagem pelo Centro Universitário de João Pessoa (UNIPE), Membro da Comissão de Controle de Infecção Hospitalar (HMDJMP), Paraíba, Brasil.

8 Graduação em Enfermagem (FESVIPE); Especialização Auditoria em serviços de Saúde (CBPEX). 


\section{Abstract}

Community infection, despite not being a major concern for health agencies, is a major concern for hospitals, as it can be a risk to already frail patients who come to health services. Therefore, the present study intends to describe the clinical profile and the prevalence of community infections of children residing in Vale do Mamanguape - PB, who were admitted to the pediatrics at the Hospital Geral de Mamanguape - PB. The methodology is a retrospective, exploratory, descriptive study with a quantitative approach. Data were extracted from electronic and printed medical records of children hospitalized for a period of more than 24 hours, between the months of January 2016 to October 2017, in the pediatric sector of the hospital. The sample included 711 children, of both sexes, aged from 0 to 15 years. The diagnosis of community infection was based on filling out the medical record, in the fields: reason for admission and anamnesis. Data were submitted to the $\mathrm{R}$ software, where descriptive and inferential data analysis was performed. In the inferential analysis, the non-parametric Pearson's Chi-Square Test was applied in order to verify the correlation between the dependent variable and the factor variables. Thus, it was observed that $83.5 \%$ children were hospitalized for some type of community infection and $16.5 \%$ were hospitalized with no infection, the average number of hospitalization days was 4.94 days $44.5 \%$ of the children hospitalized were from the municipality of Mamanguape and $55.3 \%$ from the other municipalities that make up the Vale do Mamanguape. The most frequent infections were of the respiratory tract $47.11 \%$ and gastroenteritis $14.3 \%$. Male children spent more days in hospital than female children with $\mathrm{p}$ value $=0.020$. It can be concluded that community infections are the most common causes of hospitalization in pediatrics at the Hospital Geral de Mamanguape-PB, respiratory tract infections are the most common in children living in Vale do Mamanguape.

Keywords: Infection diagnoses. Acquired infection. Infection in Children.

\section{Resumen}

La infección comunitaria, a pesar de no ser una preocupación importante para las agencias de salud, es una preocupación importante para los hospitales, ya que puede ser un riesgo para los pacientes ya frágiles que acuden a los servicios de salud. Por tanto, el presente estudio pretende describir el perfil clínico y la prevalencia de infecciones comunitarias de los niños residentes en Vale do Mamanguape - PB, que fueron ingresados en pediatría en el Hospital Geral de Mamanguape - PB. La metodología es un estudio descriptivo, exploratorio, retrospectivo con enfoque cuantitativo. Los datos fueron extraídos de historias clínicas electrónicas e impresas de niños hospitalizados por un período de más de 24 horas, entre los meses de enero de 2016 a octubre de 2017, en el sector pediátrico del hospital. La muestra incluyó a 711 niños, de ambos sexos, de 0 a 15 años. El diagnóstico de infección comunitaria se basó en el llenado de la historia clínica, en los campos: motivo de ingreso y anamnesis. Los datos se enviaron al software $\mathrm{R}$, donde se realizó un análisis de datos descriptivo e inferencial. En el análisis inferencial se aplicó la prueba no paramétrica Chi-Square de Pearson para verificar la correlación entre la variable dependiente y las variables factoriales. Así, se observó que el $83,5 \%$ de los niños fueron hospitalizados por algún tipo de infección comunitaria y el 16,5\% fueron hospitalizados sin contagio, el promedio de días de hospitalización fue de 4,94 días $44,5 \%$ de los niños hospitalizados eran del municipio de Mamanguape y el 55,3\% de los demás municipios que componen el Vale do Mamanguape. Las infecciones más frecuentes fueron del tracto respiratorio $47,11 \%$ y gastroenteritis $14,3 \%$. Los niños varones pasaron más días en el hospital que las niñas con un valor de $p=0,020$. Se puede concluir que las infecciones comunitarias son las causas más comunes de hospitalización en pediatría en el Hospital Geral de Mamanguape-PB, las infecciones del tracto respiratorio son las más comunes en los niños residentes en Vale do Mamanguape.

Palabras clave: Diagnósticos de infección. Infección adquirida. Infección en niños.

\section{Résumé}

L'infection communautaire, bien qu'elle ne soit pas une préoccupation majeure pour les agences de santé, est une préoccupation majeure pour les hôpitaux, car elle peut constituer un risque pour les patients déjà fragiles qui se rendent dans les services de santé. Par conséquent, la présente étude vise à décrire le profil clinique et la prévalence des infections communautaires des enfants résidant à Vale do Mamanguape $\mathrm{PB}$, qui ont été admis en pédiatrie à l'Hôpital Geral de Mamanguape - PB. La méthodologie est une étude rétrospective, exploratoire, descriptive avec une approche quantitative. Les données ont été extraites des dossiers médicaux électroniques et imprimés des enfants hospitalisés pour une période de plus de 24 heures, entre les mois de janvier 2016 à octobre 2017, dans le secteur pédiatrique de l'hôpital. L'échantillon comprenait 711 enfants, des deux sexes, âgés de 0 à 15 ans. Le diagnostic d'infection communautaire reposait sur le remplissage du dossier médical, dans les champs: motif d'admission et anamnèse. Les données ont été soumises au logiciel $R$, où l'analyse des données descriptives et inférentielles a été effectuée. Dans l'analyse inférentielle, le test du chi carré de Pearson non paramétrique a été appliqué afin de vérifier la corrélation entre la variable dépendante et les variables factorielles. Ainsi, il a été observé que $83,5 \%$ des enfants étaient hospitalisés pour un certain type d'infection communautaire et $16,5 \%$ étaient hospitalisés sans infection, le nombre moyen de jours d'hospitalisation était de 4,94 jours $44,5 \%$ des enfants hospitalisés étaient de la municipalité de Mamanguape et $55,3 \%$ de les autres municipalités qui composent la Vale do Mamanguape. Les infections les plus fréquentes étaient des voies respiratoires $47,11 \%$ et les gastro-entérites $14,3 \%$. Les enfants de sexe masculin ont passé plus de jours à l'hôpital que les enfants de sexe féminin avec une valeur $p=0,020$. On peut conclure que les infections communautaires sont les causes les plus courantes d'hospitalisation en pédiatrie à l'Hôpital Géral de Mamanguape$\mathrm{PB}$, les infections des voies respiratoires sont les plus courantes chez les enfants vivant à Vale do Mamanguape.

Mots-clés: Diagnostics d'infection. Infection acquise. Infection chez les enfants. 


\section{Introdução}



As IC's causam grandes impactos à humanidade tendo em vista as altas taxas de morbidade e mortalidade. Os riscos para o desenvolvimento das infecções podem estar ligados aos fatores sociais, econômicos e ambientais. Com isso, as infecções são consideradas um desafio para humanidade, instigando o desenvolvimento de especialistas, políticas e qualificação de profissionais de saúde e por organizações em busca de prevenções e controle das infecções (HENDERSON, 2013; VALLE, 2013; OLIVEIRA, 2015). A etiologia dos microrganismos das infecções comunitárias pode estar ligada a vários fatores, sendo eles endógenos do próprio organismo humano como baixo peso ao nascer, ausência no aleitamento materno, vacinação incompleta e exógeno do ambiente externo, as causas externas podem estar ligadas a: moradia em lugares precários e insalubres, limitação em acesso de informação (baixa escolaridade), permanência em creches e considerando um dos principais fatores, locais sem saneamento básico (HENDERSON, 2013; OLIVEIRA, 2015; WHITE, 2012).

As infecções comunitárias do trato respiratório são as maiores responsáveis pelos casos de morbimortalidade em crianças a nível global, em países menos desenvolvidos as infecções respiratórias são responsáveis por aproximadamente $40 \%$ dos motivos de internação de crianças e responsável pela morte de aproximadamente $20 \%$ das crianças no mundo (OMS, 2012).

Os diagnósticos das infecções podem ser baseados por vários fatores, observação clínica, testes, resultados laboratoriais entre outros. A observação direta do paciente também é uma forma de diagnosticar podendo iniciar uma terapia antimicrobiana desde que, o paciente seja acompanhado pela observação médica. Os dados coletados por testes e resultados laboratoriais servem para ampliar e diagnosticar de forma mais precisa a infecção, como identificar o patógeno e qual fármaco utilizar. Por esses motivos sempre deve-se buscar diagnosticar e tratar as infecções utilizando dados laboratoriais, de forma empírica, apenas na ausência desses dados (GARNER, 1988).

As infecções comunitárias são causadas por bactérias e vírus e nos primeiros anos de vida de um indivíduo é muito difícil definir quais desses microrganismos causam a infecção e qual é uma coinfecção, destacase na literatura a importância de seguir um cronograma de imunização para criança nos primeiros anos de vida a exemplo da vacinação contra o Haemophilus influenzae tipo b (Hib) e Streptococcus pneumoniae (ESPOSITO, 2013; ONU, 2014).

O objetivo deste estudo é caracterizar o perfil clínico e prevalência das infecções comunitária em crianças que foram internadas na pediatria do Hospital Geral de Mamanguape - PB (HGM) que residem no Vale do Mamanguape - PB. Identificar os tipos de infecções comunitária que causam a internação na pediatria e proporciona informações que possam auxiliar políticas voltadas para a prevenção de infecções comunitárias, com finalidade de evitar cada vez mais a internação desses pacientes.

\section{Materiais e Métodos}

Trata-se de um estudo retrospectivos de caráter exploratório descritivo explicativo com objetivo de uma abordagem quantitativa. A pesquisa foi realizada no Hospital Geral de Mamanguape - PB, a população estimada do município de Mamanguape é de 44.694 habitantes, localizado no Litoral Norte do Estado da Paraíba (IBGE, 2019). O cultivo da canade-açúcar faz parte da construção socioeconômica e 
socioambiental do município SANTOS, 2011; MARIZ, 2003).

O HGM é uma instituição Pública Estadual que começou suas atividades no ano de 2014, sob administração interna do Instituto de Psicologia Clínica, Educacional e Profissional (IPCEP). 0 hospital contém 70 leitos, 10 são direcionados para a internação pediátrica e os demais estão divididos entre observação da urgência, internação adulta, serviço obstetrício e Unidade de Terapia Intensiva (UTI). São realizadas também cirurgias seletivas e cardíacas pediátricas em parceria com o projeto ciclo do coração (GOVERNO DO ESTADO DA PARAÍBA, 2017).

O Hospital presta serviços também para a população de municípios circunvizinhos que compõem o Vale do Mamanguape sendo eles: Baia da Traição, Cuite de Mamanguape, Curral de Cima, Itapororoca, Jacaraú, Mamanguape, Marcação, Mataraca, Lagoa de Dentro, Pedro Regis e Rio Tinto. De 2015 a 2016 foram feitos mais de 160 mil atendimentos e mais de 1300 mil e trezentos partos realizados no ano de 2016 (GOVERNO DO ESTADO DA PARAÍBA, 2017).

A localização do município e das cidades vizinhas pode ser vista na Figura 1.

Os dados coletados para o desenvolvimento da pesquisa são referentes ao período de janeiro de 2016 a outubro de 2017. As características consideradas para os pacientes entrarem na pesquisa foram: crianças internadas na pediatria do hospital de Mamanguape - PB, de ambos os sexos com idade de 0 ano a 15 anos, contabilizando e considerando internações com tempo maior que 24 horas, estar com o prontuário legível. Foram excluídos da pesquisa, prontuários com ausência de informações como: motivo de internação, tempo de internação, pacientes que não passaram de 24 horas internados, pacientes internados fora do recorte temporal selecionado pela pesquisa. Os dados foram coletados de: relatório de pacientes internados, o qual fica armazenado no sistema de gerenciamento de dados do hospital; prontuários impressos; instrumentos de registro de informação interna assistencial e administrativo, com o insucesso dessas informações, será considerado dados insuficientes do paciente para a pesquisa.

Foi contabilizado apenas a primeira informação de internação do paciente no hospital, por ventura o paciente receber alta e por algum motivo voltasse ao mesmo setor durante o período selecionado da pesquisa, esse paciente não foi mais contabilizado na população do estudo, considerando apenas o primeiro momento de internação do paciente dentro do período estimado.

\subsection{Variável desfecho/dependente}

A IC "é aquela constatada ou em incubação no ato de admissão do paciente, desde que não relacionada com internação anterior no mesmo hospital" (BRASIL, 1998). A infecção comunitária foi reconhecida pelos mesmos critérios estabelecidos do Ministério da Saúde. A variável (desfecho) foi classificada como dicotômica, a presença foi representada pelo número 1 (um), ausência foi representada pelo número 0 (zero).

\subsection{Variável fatores}

As variáveis fatores selecionadas foram, sexo, idade, localidade, motivo de internação e tempo de internação.

\subsection{Inferência estatística}

$\mathrm{Na}$ análise estatística dos dados foi utilizado o software estatístico R, processando os dados no teste qui-quadrado de Pearson, considerando o teste como significativo com $p$-valor menor que 5\% (pvalor $<0,05)$. 0 teste qui-quadrado de Pearson pode ser utilizado para encontrar diferenças entre grupos observados, tendo em vista um número esperado ou resposta baseando-se em hipóteses (SIEGEL, 2006).

A pesquisa utiliza considerações orientadas pela resolução 466/2012 que regulamenta pesquisa envolvendo seres humanos, a qual preconiza a afirmação pela dignidade e liberdade. 0 estudo dispensa o Termo de Consentimento Livre Esclarecido (TCLE) pois os dados trabalhados foram dados secundários de prontuários sem interferir no tratamento de pacientes. 0 estudo tem a aprovação do Comitê de Ética em Pesquisa da Universidade Federal da Paraíba com justificativa da dispensa do (TCLE), sob número de parecer 2.410.341.

\section{Resultados}

Durante o período de janeiro de 2016 a outubro de 2017 foram contabilizados 804 pacientes que 
passaram mais de 1 dia internados na pediatria do Hospital Geral de Mamanguape- PB. A amostra consistiu em $711(88,43 \%)$ crianças os quais apresentaram todas as informações necessárias para o presente estudo. Sendo $350(49,3 \%)$ do sexo feminino e 361 (50,7\%) do sexo masculino. A (Figura 1) ilustra a distribuição de presença e ausência de IC. Considerando apenas o grupo de crianças do sexo feminino, 55 crianças (16\%) foram internadas sem nenhuma IC e 295 (84\%) foram internadas devido à algum tipo de IC. Ao analisar apenas o grupo de crianças do sexo masculino, $61(16,8 \%)$ foram internadas sem nenhuma infecção comunitária e 300 $(83,2 \%)$ foram diagnosticadas com algum tipo de IC.

A variável idade, a menor idade registrada foi de 0 ano e maior idade de 15 anos, com média de idade dos pacientes internados de 3 anos. 0 menor dia registrado foi de 1 (um) dia, a média de dias de internação atingiu 4,94 e a maior quantidade de dias de internação registrado na pediatria foi de 25 dias. No tocante da localidade, o município com maior número de pacientes internados no período do estudo foi o município de Mamanguape - PB com 317 $(44,6 \%)$ pacientes, em seguida o município de Rio Tinto com 85 (12\%), Marcação 54 (7,6\%), Capim 52 (7,3\%), Baia da Traição com 50 (7\%), Itapororoca 44 $(6,2 \%)$, Jacaraú $35(4,9 \%)$, Cuité de Mamanguape 30 $(4,2 \%)$, Mataraca $19(2,7 \%)$, Pedro Regis $8(1,1 \%)$, Curral de Cima $8(1,1 \%)$, Araçagi $1(0,1 \%)$ e Outros 8 $(1,1 \%)$ dos pacientes.

A Tabela 2, destaca o quantitativo de pacientes internados com IC, tipos IC e pacientes sem a presença de IC. Observa-se que, os motivos de internação mais frequentes na pediatria do HGM no período de janeiro de 2016 a outubro de 2017, foi infecção do trato respiratório, com total 335 $(47,11 \%)$. A segunda maior causa de internação não se refere às infecções e sim por motivos adversos como: quedas, intoxicação entre outros. A gastroenterite foi o segundo maior tipo de IC 102 $(14,3 \%)$, em sequência ficaram Infecções cutâneas 49 $(6,89 \%)$, Infecção do trato urinário 40 (5,62\%) entre os outros tipos de IC.

Foi utilizando o teste qui-quadrado, com evidência estatística significativa com ( $p$-valor $=0,02$ ) que existe diferença entre os grupos do sexo feminino e masculino na quantidade de dias de internação (Tabela 3).

Para os dias de internação foram divididos dois grupos. $1^{\circ}$ grupo crianças que passaram cinco ou mais de cinco dias internadas e $2^{\circ}$ grupo crianças que passaram menos de cinco dias. Correlacionando os grupos com idade igual ou superior a 1 ano de idade. As crianças que passaram mais de cinco dias internadas foram crianças com idade igual ou superior de 1 ano de idade, existindo evidencia estatísticas significativa com $p$-valor $=0,005$ que existe associação entre essas variáveis, para crianças que ficaram internadas por mais de 5 dias são geralmente maiores de 1 ano de idade (Tabela 3 ).

\section{Discussão}

As Infecções Respiratórias Agudas (IRA) foram as mais frequentes entre as infecções comunitária, as quais são consideradas infecções do trato respiratório, significando as mais presentes nos motivos de internação no HGM. Esse resultado é reforçado por outros estudos que as infecções do trato respiratório são as mais frequentes nos serviços hospitalares, variando entre bronquiolite $e$ pneumonia dependendo do critério de diagnóstico que definem as doenças MARTINS, 2016; PASSOS, 2018; BRASIL, 2012). A forma de avaliação médica utilizada para diagnosticar as IC do trato respiratório no HGM foram através da avaliação clínica e diagnostico radiológico.

As infecções comunitárias representadas por doenças respiratórias em crianças podem ser influenciadas por uma complexidade de vários motivos no ambiente em que vivem, presença de fumantes de tabaco no ambiente familiar, fumaças e queimas de algum tipo de combustível de cozinha, partículas aerossóis ao ar livre, fungos de ambiente interno das casas, águas contaminadas entre outros. Todos esses pontos citados são geralmente encontrados no ambiente familiar, ambiente este, onde as crianças passam por mais tempo principalmente em seus primeiros anos de vida ALDOUS, 1996).

Para as gastroenterites a qual foi o segundo maior motivo de internação por IC no estudo, conforme a Tabela 2. Existem aproximadamente dois bilhões de casos de doenças diarreicas no mundo, todos os anos, 1,9 milhão de crianças com menos de cinco anos de idade sofrem de diarreia a cada ano (FARTHING, 2012; KENNELEY 2010). A gastroenterite é uma das causas mais frequentes de morbidade e mortalidade em crianças, e está entre os maiores motivos de atendimento de serviço hospitalar, entre os serviços 
hospitalares estão as internações no setor de pediatria (EPIFANIO, 2018).

Além de fatores socioambientais e socioeconômicos que podem aumentar as IC, é importante elucidar o mau uso de antimicrobianos que acarreta em resistência bacteriana sobre o antibiótico utilizado fora do ambiente hospitalar. A início os cuidadores dos pacientes com IC buscam os serviços primários de saúde os quais geralmente não existem uma comissão ou ficha de controle de vigilância para uso de antimicrobianos apenas receita médica. Ao contrário dos serviços hospitalares, os quais instalam comissões de vigilância através da RDC 2616/98 legislação específica para instalação, implantação e funcionamento, e mesmo assim, com esse controle no ambiente hospitalar ainda existe o uso indiscriminado (LIMA, 2018).

Foi elucidado na Tabela 3 , que crianças do sexo masculino passam mais tempo internadas na pediatria do que crianças do sexo feminino ( $p$-valor= $0,005)$. A relação do sexo e dias de internação foi estatisticamente significante. A Tabela 3 demonstra também que crianças em seus primeiros anos de vida passam mais de 5 dias internadas, podendo ser considerado como fatores de imaturidade do seu sistema imunológico conforme a literatura (CARVALHO, 2018).

Famílias de baixa renda com crianças abaixo de dois anos são considerados grupos de mais riscos para desenvolver IC, sobretudo para alguns tipos de infecções os pais têm a percepção de que são doenças comuns na infância de seus filhos e geralmente buscam medicar seus filhos antes mesmo de buscar um serviço hospitalar. A exemplo de crianças que desenvolve as IRAs (PASSOS, 2018).

Houve limitações referente aos dados secundário, como registros incompletos nos prontuários e ausência de cultura laboratorial bacteriana das crianças internadas na pediatria, impossibilitando a identificação dos microrganismos causadores da IC. Não constatou cultura de admissão dos pacientes internados na pediatria do HGM, na instituição é mais comum fazer cultura de admissão de pacientes internados com mais frequência na UTI ou em clínica médica através de solicitação médica.

Analisar o perfil dos pacientes internados e utilizar esses resultados para aperfeiçoar e especializar os serviços de internação pediátrica será a maior serventia dessa pesquisa. 0 estudo permite também, que possamos refletir em aprimoramento e melhorar os serviços prestados nesse setor. Será importante avaliar se há viabilidade e se cabe fortalecer os mecanismos de diagnósticos, a exemplo de implementação de IC mais frequentes nos pacientes internados.

Com base nos resultados do estudo, houve uma alta prevalência de crianças internadas na pediatria por motivos de infecção comunitária, alcançando $82 \%$. Foi diagnosticado um maior número de infecções do trato respiratório representando $47,11 \%$ entre os outros tipos de infecções. Foi comprovado também que crianças do sexo masculino passam mais tempo internados do que crianças do sexo feminino na pediatria do HGM.

\section{Considerações Finais}

Pode-se concluir que o resultado ilustrado condiz com a literatura quando se refere às infecções respiratórias, as quais são mais cumulativas entre as infecções comunitárias, evidenciando a necessidade de melhores condições de vida do ambiente em que vive essa população, sobretudo condições ambientais de saneamento, bem como aprimorar o serviço básico de saúde e preventivo.

\section{REFERENCIAS}

ALDOUS, M.B.; HOLBERG C.J.; WRIGHT, A.L.; MARTINEZ F.D.; TAUSSIG, L.M. Evaporative cooling and other home factors and lower respiratory tract illness during the first year of life. American journal of epidemiology, v. 143, n. 5, p. 423-430, 1996.

BRASIL. Ministério da Saúde. Diário Oficial da União, Portaria número 2616 de 12 de maio de 1998 [Internet]. Brasília, 1998 [cited 2017 March 16]. Disponível em: http://bvsms.saude.gov.br/bvs/saudelegis/gm/199 8/prt2616_12_05_1998.html.

Instituto Brasileiro de Geografia e Estatística. IBGE cidades. 2019 [cited 2020 Jul 14]. Disponível em: https://cidades.ibge.gov.br/brasil/pb/mamanguap e.

- Conselho Nacional de Saúde. Resolução número 466, de 12 de dezembro de 2012 [cited 2018 Jul 04]. Brasília. Disponível em: http://www.conselho.saude.gov.br/web_comissoes /conep/index.html. 
CARVALHO, F.C. Epidemiologia descritiva da Síndrome Respiratória Aguda Grave pelo vírus sincicial respiratório no Brasil, 2014 - 2017 (até SE 32) [monography]. [Brasília]: Faculdade de Ciências da Educação e Saúde; 21p. 2017.

EPIFANIO, M.; PORTELA, J.D.L.; PIVA, J.P.; FERREIRA, C.H.T.; SARRIA, E.E.; MATTIELLO, R. Bromopride, metoclopramide, or ondansetron for the treatment of vomiting in the pediatric emergency department: a randomized controlled trial. Jornal de pediatria, v. 94, p. 62-68, 2018.

ESPOSITO, S.; ZAMPIERO, A.; TERRANOVA, L.; IERARDI, V.; ASCOLESE, B.; DALENO, C.; PRINCIPI, N. Pneumococcal Bacterial Load Colonization as a Marker of Mixed Infection in Children With Alveolar Community-acquired Pneumonia and Respiratory Syncytial Virus or Rhinovirus Infection. The Pediatric infectious disease journal, v. 32 , n. 11, p. 11991204, 2013.

FARTHING, M.; SALAM, M. A.; LINDBERG, G.; DITE, P.; KHALIF, I.; SALAZAR-LINDO, E.; LEMAIR, A. Acute diarrhea in adults and children: a global perspective. Journal of clinical gastroenterology, v. 47, n. 1, p. 12-20, 2013.

GARNER, J.S.; JARVIS, W.R.; EMORI, T.G.; HORAN, T.C.; HUGHES, J.M. CDC definitions for nosocomial infections, 1988. American journal of infection control, v. 16, n. 3, p. 128-140, 1988.

HENDERSON, K.L.; MÜLLER-PEBODY, B.; JOHNSON, A.P.; WADE, A.; SHARLAND, M.; GILBERT, R. Community-acquired, healthcare-associated and hospital-acquired bloodstream infection definitions in children: a systematic review demonstrating inconsistent criteria. Journal of Hospital Infection, v. 85, n. 2, p. 94-105, 2013.

KENNELEY, I.L. Infection control and the home care environment. Home Health Care Management \& Practice, v. 22, n. 3, p. 195-201, 2010.

LIMA, H.K.S; CARVALHO, H.E.F.; SOUSA, A.F.L.; MOURA, M.E.B.; ANDRADE, D.; VALLE, A.R.M.C. Distribuição e custo de antimicrobianos na Atenção Primária. Acta Paulista de Enfermagem, v. 31, n. 1, p. 95-101, 2018.
MARIZ, C. Mamanguape: passado e presente-1943. In: Ramos JO. Pedaços da História de Mamanguape. João Pessoa: Persona. 2003.

MARTINS, A.L.O.; NASCIMENTO, D.S.F.; SCHNEIDER, I.J.C.; SCHUELTER-TREVISOL, F. Incidência de infecções comunitárias de vias aéreas inferiores em crianças. Revista Paulista de Pediatria, v. 34, p. 204-209, 2016.

OLIVEIRA, B.R.G.; VIERA, C.S.; FURTADO, M.C.C.; MELO, D.F.; LIMA, R.A.G. Perfil de morbidade de crianças hospitalizadas em um hospital público: implicações para a Enfermagem. Revista Brasileira de Enfermagem, v. 65, p. 586-593, 2012.

PASSOS, S.D.; MAZIERO, F.F.; ANTONIASSI, D.Q.; SOUZA, L.T.D.; FELIX, A.F.; DOTTA, E.; GAZETA, R.E. Doenças Respiratórias Agudas em crianças brasileiras: os cuidadores são capazes de detectar os primeiros sinais de alerta? Revista Paulista de Pediatria, v. 36, p. 3-9, 2018.

SANTOS, J.S. História e Desenvolvimento Regional em Cidades da Paraíba: Construção para o desenvolvimento histórico do 37 Estado. Campina Grande: Cópias e Papéis. 2011.

SES-PB. Secretaria de Saúde do Estado da Paraíba. Hospital Geral de Mamanguape - PB [Internet]. 2017 [cited 2017 Jun 12]. Disponível em: http://paraiba.pb.gov.br/hospital-geral-demamanguape-registra-mais-de-6-mil atendimentosem-maio/.

SOUSA, A.F.L.; QUEIROZ, A.A.F.L.N.; OLIVEIRA, L.B.; VALLE, A.R.M.C.; MOURA, M.E.B. Social representations of community-acquired infection by primary care professionals. Acta Paulista de Enfermagem, v. 28, p. 454-459, 2015.

SIEGEL, S.; CASTELLAN, N.J. Estatística NãoParamétrica para Ciências do Comportamento. 2a ed. Porto Alegre: Artmed, 2006.

UNICEF. United Nations Children's Fund. Every child counts : revealing disparities, advancing children's rights. 2014 [cited 2018 Jul 14]. Disponível em: https://www.unicef.org/gambia/SOWC_report_201 4.pdf 
VALLE, A.R.M.C. Competências do enfermeiro para ações preventivas na atenção domiciliar com ênfase nos riscos de infecção. Tese de doutorado. 262 p. São Paulo: USP, 2013.

WARDLAW, T.M.; JOHANSSON, E.W.; HODGE, M. Pneumonia: the forgotten killer of children. Geneva: World Health Organization; 2006.
WHITE, R.; SWALES, B.; BUTCHER, M. Principles of infection management in community-based burns care. Nursing Standard, v. 27, n. 2, p. 64, 2012. 